Bangladesh J. Bot. 39(2): 169-177, 2010 (December)

\title{
INDIGENOUS KNOWLEDGE OF HERBAL MEDICINES IN BANGLADESH. 3. TREATMENT OF SKIN DISEASES BY TRIBAL COMMUNITIES OF THE HILL TRACTS DISTRICTS
}

\author{
Mohammad Atiqur Rahman \\ Department of Botany, University of Chittagong, Chittagong-4331, Bangladesh
}

Key words: Indigenous knowledge, Herbal medicines, Skin diseases, Tribes, Bangladesh

\begin{abstract}
Indigenous knowledge of herbal medicines for skin diseases like boils, eczema, scabies, septic abscess, itching and skin allergy, burns, chicken pox, warts and leucoderma, fungal and bacterial infections, including healing cuts and wounds has been documented by randomly interviewing Chakma, Marma and Tanchunga tribes of the Hill Tracts districts of Bangladesh since 1995. The plant parts of 60 species belonging to 40 families were known to be used. The plant specimens were collected, identified and preserved at the Herbarium of Chittagong University. An enumeration of these plants is presented along with their tribal names, categories of skin diseases, plant parts with preparation and route of administration.
\end{abstract}

\section{Introduction}

The Hill Tracts of Bangladesh includes three districts, viz., Bandarban, Khagrachari and Rangamati, and are located in the south east corner of the country with Kaptai Watershed area between $21^{\circ} 25^{\prime}$ and $23^{\circ} 45^{\prime} \mathrm{N}$ and between $91^{\circ} 45^{\prime}$ and $92^{\circ} 52^{\prime} \mathrm{E}$.

A large number of tribal populations under 14 major tribes, viz., Chakma, Marma, Murong, Tanchunga, Tripura, Chack, Bhome, Pangkhoa, Kheyang, Rheyang, Rakhain, Lushai, Kuki and Khumi live as forest inhabitants in the remote areas throughout the Hill Tracts where there is no or poor introduction of education and modern medical systems of health care (Rahman et al. 2003a). A vast knowledge of use of plants as sources of medicine was known to be available among elderly men and women, specially among traditional herbalists who are known as baiddaya. This valuable indigenous knowledge is disappearing day by day due to the development of modern health care systems in the hilly areas (Rahman et al. 2003b). The documentation and publications on the indigenous knowledge or the use of plants by tribal people are meagre (Alam 1992, Rahman 1997, 1999, 2007, Rahman and Uddin 1998, Rahman et al. 1998, 2003a, 2003b, 2007).

Preparation of a complete inventory of this indigenous knowledge of herbal medicine are in progress under the Biodiversity Link Project between Chittagong (Bangladesh) and Aberdeen (UK) Universities since 1995. The first report of the series was made on the herbal treatment of Jaundice (Rahman et al. 2003a) and the second report was on the cure of diarrhoea, dysentery, indigestion and stomachace (Rahman et al. 2003b). The present communication, the third of the series, deals with the indigenous knowledge of treating skin diseases among three major tribes, Chakma, Marma and Tanchunga of the Hill Tracts districts of Bangladesh.

\section{Materials and Methods}

Documentation of the knowledge of herbal practices for the cure of skin diseases including healing cuts and wounds of skin, was made under Biodiversity Link Project among three major tribes, Chakma, Marma and Tanchunga of Bandarban, Rangamati and Khagrachari districts, Bangladesh. Information/data were recorded by taking random interviews of the herbalists, elderly men and women. The vernacular names, methods of preparation and route of administration were

E-mail of the author: <atiquerahman125@hotmail.com> 
recorded through repeated interviews of practitioners. The source and location of plant material were traced out and recognized with the help of informers. The fertile plant samples were collected, photographed and preserved at the Herbarium of Chittagong University (HCU) as voucher specimens. The plant specimens were critically examined with the help Long-arm Stereo Microscope (LSM) and identified by comparing with the identified specimens of Bangladesh National Herbarium (DACB), and HCU and Bangladesh Forest Research Institute herbarium (BFRIH). The Flora of Bangladesh and recently published other local Floras including Ahmed et al. (2009) were also consulted for current nomenclature.

Medicinal and ethnobotanical literature such as, Chopra (1958), Chopra et al. (1956), Dastur (1977), Hassan and Huq (1993), Hassan and Khan (1986, 1996), Jain (1991), Ghani (2002), Khan et al., (2002), Mia and Huq (1988), Rahman and Uddin (1998), Rahman (1999), Yusuf et al. (1994, 2009) were consulted for pharmacological properties and ethnobotanical reports of recorded species. An enumeration of these plants is prepared with the documented information.

\section{Results and Discussions}

The ethnobotanical investigation on skin diseases among Chakma, Marma and Tanchunga tribes revealed that mostly elderly men and women are commonly suffering from eczema, scabies, septic abscess, fungal/bacterial infection, boils, wounds and skin allergy. More than $70 \%$ of them receive herbal treatments from either Baiddaya or elderly women. It was revealed that mostly Chakma tribe of Rangamati district have their vast knowledge of herbal treatments compared to other tribes, and that the herbal treatments still remain as an integral part of the health care systems. The documented data also revealed that the bacterial and fungal infections, eczema and skin allergy are more common and herbal medicine is usually the means of treatment.

Screening of the voucher specimens resulted in the identification of 60 plant species of which 25 are used for the treatment of boils, 10 for allergy and itching, 9 for fungal/bacterial infections, 8 for healing cuts and wounds, 6 for each of eczema and scabies, 5 for septic abscess, 1 for each of wart, acne, leucoderma, chicken pox and measles. Twelve species are found to have been using for the treatment of more than one category, and Cardiospermum helicacabum L. is remarkably used by almost all tribes for the treatment of chicken pox. The medicinal literature, so far reviewed, revelaled that none of these recorded species has its pharmacological properties against skin ailments. However, the plants used for the treatment of several categories of skin diseases are enumerated in Table 1.

Table 1. Enumeration of plants used for the treatment of skin diseases by tribes. (Abbreviations: $\mathrm{R}$, Rangamati; K, Khagrachari and B, Bandarban; C, Chakma; M, Marma and T, Tanchunga).

\begin{tabular}{|c|c|c|c|c|}
\hline $\begin{array}{l}\text { Botanical name, Family/ Voucher } \\
\text { number and area of collection }\end{array}$ & $\begin{array}{l}\text { Tribal names } \\
\text { of plants }\end{array}$ & $\begin{array}{l}\text { Disease } \\
\text { category }\end{array}$ & $\begin{array}{l}\text { Plant } \\
\text { parts }\end{array}$ & $\begin{array}{l}\text { Preparation and route } \\
\text { of administration }\end{array}$ \\
\hline $\begin{array}{l}\text { 1. Abelmoschus moschatus Medik. } \\
\text { Malvaceae/ L2961: K; Fig. } 16 \\
\text { 2. Thladiantha cordifolia (Blume) } \\
\text { Cogn. Cucurbitaceae/ L3148: K }\end{array}$ & $\begin{array}{l}\text { Konigaas (C) } \\
\text { Paranga ludi } \\
\text { (C) }\end{array}$ & Wounds & leaf & $\begin{array}{l}\text { Fresh leaves of both plants } \\
\text { crushed, made into paste } \\
\text { and applied }\end{array}$ \\
\hline $\begin{array}{l}\text { 3. Achyranthes aspera L. } \\
\text { Amaranthaceae/ L1820: K; Fig. } 20\end{array}$ & $\begin{array}{l}\text { Upolengra, } \\
\text { Ublengra }(\mathrm{C})\end{array}$ & Boils & leaf & $\begin{array}{l}\text { Fresh leaf-paste applied to } \\
\text { the affected areas }\end{array}$ \\
\hline $\left.\begin{array}{l}\text { 4. Allophyllus triphyllus (Burm. f.) } \\
\text { Merr. Sapindaceae/ L3421: R } \\
\text { 5. Azadirachta indica A. Juss. } \\
\text { Meliaceae/ L4046a: R }\end{array}\right\}$ & $\begin{array}{l}\text { Dhendrema, } \\
\text { Jhandhara (C) } \\
\text { Neem (C) }\end{array}$ & Scabies & leaf & $\begin{array}{l}\text { Infusion of leaves of both } \\
\text { plants used in washing } \\
\text { infected areas twice daily } \\
\text { for 3-4 days }\end{array}$ \\
\hline
\end{tabular}




\begin{tabular}{|c|c|c|c|c|}
\hline $\begin{array}{l}\text { 6. Amomum dealbatum Roxb. } \\
\text { Zingiberaceae/ L4493: R; Fig. } 22\end{array}$ & $\begin{array}{l}\text { Palachengay } \\
\text { (C) }\end{array}$ & $\begin{array}{l}\text { Septic } \\
\text { abscess }\end{array}$ & $\begin{array}{l}\text { rhizo- } \\
\text { me }\end{array}$ & $\begin{array}{l}\text { Rhizome extract applied } \\
\text { to affected areas }\end{array}$ \\
\hline $\begin{array}{l}\text { 7. Ardisia solanacea Roxb. } \\
\text { Myrsinaceae/L3457:R ; Fig. } 9\end{array}$ & $\begin{array}{l}\text { Barochulliya } \\
\text { (C) }\end{array}$ & Boils & leaf & $\begin{array}{l}\text { Fresh leaf-paste applied } \\
\text { externally }\end{array}$ \\
\hline $\begin{array}{l}\text { 8. Asparagus racemosus L. } \\
\text { Liliaceae/ L4493c:: R }\end{array}$ & Sati-sara (C) & $\begin{array}{l}\text { Septic } \\
\text { abscess }\end{array}$ & $\begin{array}{l}\text { leaf, } \\
\text { root }\end{array}$ & $\begin{array}{l}\text { Leaf-/root-paste applied 2- } \\
3 \text { times }\end{array}$ \\
\hline $\begin{array}{l}\text { 9. Bidens sulphurea (Cav.) } \\
\text { Sch. Bip. Asteraceae/ L4000: R }\end{array}$ & Shumo phul (C) & Acne & $\begin{array}{l}\text { flower, } \\
\text { leaf }\end{array}$ & $\begin{array}{l}\text { Flower and leaf paste and } \\
\text { applied to affected areas }\end{array}$ \\
\hline $\begin{array}{l}\text { 10. Boehmeria nivea } \\
\text { Urticaceae/ L4493b: R }\end{array}$ & $\begin{array}{l}\text { Hurumbuto- } \\
\text { pada }(\mathrm{C})\end{array}$ & $\begin{array}{l}\text { Wound, } \\
\text { septic } \\
\text { abscess }\end{array}$ & leaf & $\begin{array}{l}\text { Leaf-paste applied twice a } \\
\text { day }\end{array}$ \\
\hline $\begin{array}{l}\text { 11. Bridelia retusa (L.) Spreng } \\
\text { Euphorbiaceae/ L4481: R }\end{array}$ & $\begin{array}{l}\text { Shukujja gaas } \\
\text { (C) }\end{array}$ & $\begin{array}{l}\text { Skin } \\
\text { infection }\end{array}$ & leaf & $\begin{array}{l}\text { Leaf-paste applied to } \\
\text { affected areas }\end{array}$ \\
\hline $\begin{array}{l}\text { 12. Bridelia stipularis (L.) Blume } \\
\text { Euphorbiaceae/ L3143: K; Fig. } 13\end{array}$ & $\begin{array}{l}\text { Bangari } \\
\text { bhanga gaas (C) }\end{array}$ & Allergy & leaf & $\begin{array}{l}\text { Leaf extract given to drink } \\
\text { 2-3 times a day }\end{array}$ \\
\hline $\begin{array}{l}\text { 13. Byttneria pilosa Roxb. } \\
\text { Sterculiaceae/ L2962: K; Fig. } 10\end{array}$ & $\begin{array}{l}\text { Sola ludi, } \\
\text { Chalaludi (C) }\end{array}$ & $\begin{array}{l}\text { Boils, } \\
\text { Scabies }\end{array}$ & $\begin{array}{l}\text { stem, } \\
\text { leaf }\end{array}$ & $\begin{array}{l}\text { Stem-paste applied to } \\
\text { boils; infusion of leaves } \\
\text { used in baths for scabies }\end{array}$ \\
\hline $\begin{array}{l}\text { 14. Caesalpinia bonduc (L.) Roxb. } \\
\text { Caesalpiniaceae/ 3450: R }\end{array}$ & $\begin{array}{l}\text { Kumujja ludi } \\
\text { (C) }\end{array}$ & $\begin{array}{l}\text { Skin } \\
\text { infection }\end{array}$ & leaf & $\begin{array}{l}\text { Fresh tender leaf-paste } \\
\text { applied to affected areas }\end{array}$ \\
\hline $\begin{array}{l}\text { 15. Cardiospermum helicacabum L. } \\
\text { Sapindaceae/ L1547: R; Fig. } 7\end{array}$ & $\begin{array}{l}\text { Ketha boitta } \\
\text { shag, Kedabok } \\
\text { shag (C) }\end{array}$ & $\begin{array}{l}\text { Chicken } \\
\text { pox }\end{array}$ & $\begin{array}{l}\text { whole } \\
\text { plant }\end{array}$ & $\begin{array}{l}\text { Hot water extract of whole } \\
\text { plant given to drink 2-3 } \\
\text { times a day }\end{array}$ \\
\hline $\begin{array}{l}\text { 16. Centella asiatica L. } \\
\text { Apiaceae/ L3048b: R }\end{array}$ & Mingooni (C) & Eczema & leaf & $\begin{array}{l}\text { Leaf-paste applied to the } \\
\text { twice daily }\end{array}$ \\
\hline $\begin{array}{l}\text { 17. Costus speciosus (Koenig) Sm. } \\
\text { Costaceae/ L1553: R; Fig. } 4\end{array}$ & $\begin{array}{l}\text { Ketoki, Kedogi } \\
\text { Ma laksmi ma } \\
\text { (C) }\end{array}$ & $\begin{array}{l}\text { Boils, } \\
\text { itch }\end{array}$ & leaf & $\begin{array}{l}\text { Fresh leaf-paste applied to } \\
\text { affected areas 2-3 times }\end{array}$ \\
\hline $\begin{array}{l}\text { 18. Crinum asiaticum L. Fig. } 31 \\
\text { Amaryllidaceae/ L663: R, Fig. } 27\end{array}$ & $\begin{array}{l}\text { Koba rashun } \\
\text { (C) }\end{array}$ & Boils & leaf & $\begin{array}{l}\text { Fresh root-paste applied } \\
\text { to the boils until it gets dry }\end{array}$ \\
\hline $\begin{array}{l}\text { 19. Crotalaria verrucosa L. } \\
\text { Papilionaceae/ L1314: K }\end{array}$ & $\begin{array}{l}\text { Kuduk } \\
\text { jhunjhuni (C); } \\
\text { Easygaas (T) }\end{array}$ & $\begin{array}{l}\text { Allergy, } \\
\text { Infection, } \\
\text { wounds }\end{array}$ & leaf & $\begin{array}{l}\text { Leaf extract applied to the } \\
\text { affected parts }\end{array}$ \\
\hline $\begin{array}{l}\text { 20. Cuscuta reflexa Roxb. } \\
\text { Cuscutaceae/ L5314: B }\end{array}$ & $\begin{array}{l}\text { Pargasa }(\mathrm{C}) \\
\text { Zigro }(\mathrm{M})\end{array}$ & Eczema & $\begin{array}{l}\text { whole } \\
\text { plant }\end{array}$ & $\begin{array}{l}\text { Paste of plant applied to } \\
\text { affected areas until } \\
\text { recovery }\end{array}$ \\
\hline $\begin{array}{l}\text { 21. Cyclea barbata Miers. } \\
\text { Menispermaceae/ L1737: K }\end{array}$ & $\begin{array}{l}\text { Patalpur (C), } \\
\text { Wambokhor } \\
(\mathrm{M})\end{array}$ & $\begin{array}{l}\text { Allergy, } \\
\text { itching }\end{array}$ & leaf & $\begin{array}{l}\text { Leaf extract given to } \\
\text { children for drink twice } \\
\text { daily }\end{array}$ \\
\hline $\begin{array}{l}\text { 22. Cymbidium aloifolium (L.) Sw. } \\
\text { Orchidaceae/L.: R; Fig. } 21\end{array}$ & Suri mach $(\mathrm{T})$ & Boils & leaf & $\begin{array}{l}\text { Leaf-paste applied to boils } \\
\text { twice daily }\end{array}$ \\
\hline $\begin{array}{l}\text { 23. Dendrophthoe falcata (L.f.) Etting. } \\
\text { Loranthaceae/ L. s.n.: R }\end{array}$ & $\begin{array}{l}\text { Sat-jat parsallo } \\
\text { (C) }\end{array}$ & $\begin{array}{l}\text { Itching, } \\
\text { allergy }\end{array}$ & root & $\begin{array}{l}\text { Root extract applied 2-3 } \\
\text { times a day }\end{array}$ \\
\hline
\end{tabular}


(contd.)

24. Dracaena spicata Roxb. Agavaceae/ L4470: B

25. Eclipta alba L. Asteraceae/ L1741: R; Fig. 23

26. Emblica officinalis Gaertn. Euphorbiaceae/cult.

27. Eupatorium odoratum L. Asteraceae/ L1741a: R

28. Ficus mollis Vahl. Moraceae/ L4003: R; Fig. 15

29. Holarrhena antidysenterica Wall. Apocynaceae/ L2813: R; Fig. 26

30. Hyptis suaveolens (L.) Poit. Lamiaceae/ L4470: R; Fig. 17

31. Impatiens flavida Colebr. Balsaminaceae/ L674b: R

32. Leea indica $\mathrm{L}$. Leeaceae/ L4470: R

33. Limnophila repens Benth. Scrophulariaceae/ L5428a: R

34. Mimosa pudica L. Mimosaceae/ L4024: R

35. Litsea glutinosa (Lour.) Robin.; Lauraceae; L5191: B

36. Macaranga peltata (Roxb.) Muell.- Arg. Euphorbiaceae/ L4027: R; Fig. 24

37. Maesa acuminata A. DC. Myrsinaceae/ L2461: R

38. Merremia vitifolia (Burm.f.) Hallier f.

Convolvulaceae/2795: R

39. Mikania micrantha Kunth Asteraceae/ L2342: R; Fig. 11

40. Mimosa intisia L. Fig. 12 Mimosaceae/ L3453: R

41. Smilax zeylanica $\mathrm{L}$. Smilacaceae/ L3453a: R; Fig. 29

42. Mussaenda roxburghii Hook.f. Rubiaceae/ L1535: R; Fig. 19

43. Neonauclea sessilifolia (Roxb.) Merr. Rubiaceae/ L1546: R; Fig. 1

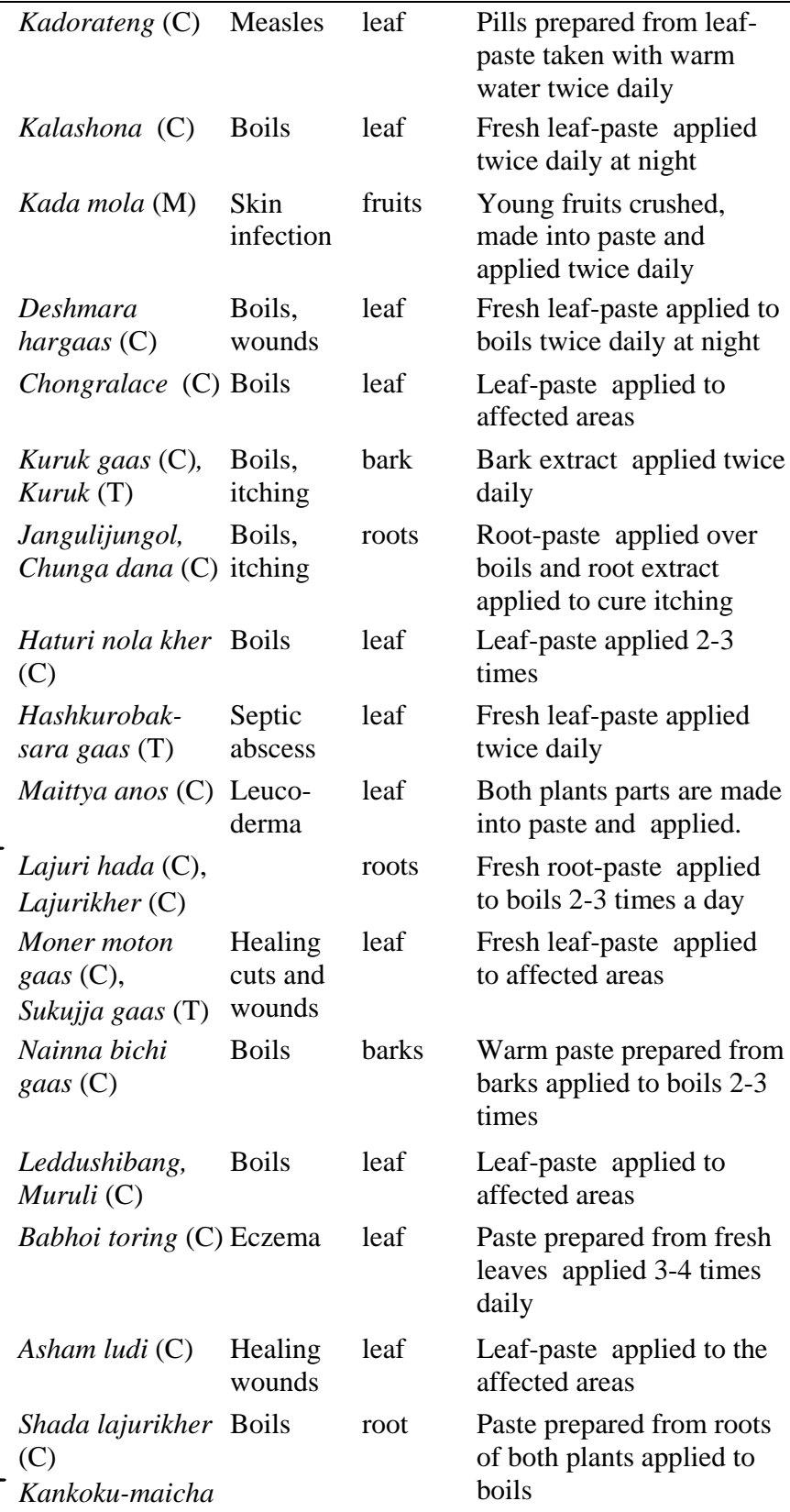
paste taken with warm water twice daily

Kalashona (C) Boils leaf

Kada mola (M) Skin fruits infection Fresh leaf-paste applied twice daily at night

Young fruits crushed, made into paste and applied twice daily

Deshmara Boils, leaf hargaas (C) wounds Fresh leaf-paste applied to

Chongralace (C) Boils leaf

Kurukgaas (C), Boils, bark Kuruk (T) itching

Jangulijungol, Boils, Chunga dana (C) itching

Haturi nola kher Boils leaf (C)

Hashkurobak- Septic leaf saragaas $(\mathrm{T})$ abscess

Maittya anos (C) Leuco- leaf

leaf Both plants parts are made into paste and applied.

Lajuri hada (C), roots

Lajurikher (C)

Moner moton Healing leaf gaas (C), cuts and Sukujja gaas (T) wounds Nainna bichi Boils barks gaas (C)

Leddushibang, Boils leaf Muruli (C)

Babhoi toring (C) Eczema leaf

Asham ludi (C) Healing leaf wounds

Shada lajurikher Boils root (C)

Kankoku-maicha boils twice daily at night

Leaf-paste applied to affected areas

Bark extract applied twice daily

Root-paste applied ove boils and root extract applied to cure itching Leaf-paste applied 2-3 times

Fresh leaf-paste applied twice daily to boils 2-3 times a day

Fresh leaf-paste applied to affected areas

Warm paste prepared from barks applied to boils 2-3 times

Leaf-paste applied to affected areas

Paste prepared from fresh leaves applied 3-4 times daily

Leaf-paste applied to the affected areas

Paste prepared from roots of both plants applied to boils (C) Ranirtak, Boils leaf Sheodima, Patto mormoijja

Kam gaas (C) Skin leaf Infection
Fresh leaf-paste applied to boils

Fresh leaf-paste applied twice daily 


\begin{tabular}{|c|c|c|c|c|}
\hline $\begin{array}{l}\text { 44. Peristylus constrictus (Lindl.) } \\
\text { Lindl. Orchidaceae/L5180: R }\end{array}$ & Bhuinora (T) & Boils & root & $\begin{array}{l}\text { Root-paste applied to } \\
\text { boils 2-3 times a day }\end{array}$ \\
\hline $\begin{array}{l}\text { 45. Plumbago indica L. } \\
\text { Plumbaginaceae/ L5086: R }\end{array}$ & Aguni tita (C) & Scabies & leaf & $\begin{array}{l}\text { Leaf-paste applied to } \\
\text { affected areas }\end{array}$ \\
\hline $\begin{array}{l}\text { 46. Pouzolzia sanguinea (Blume) Merr } \\
\text { Urticaceae/ L1712a: K; Fig. } 3\end{array}$ & $\begin{array}{l}\text { Mogjangaillya } \\
\text { shak (T) }\end{array}$ & $\begin{array}{l}\text { Skin } \\
\text { infection }\end{array}$ & leaf & $\begin{array}{l}\text { Leaf extract applied for } \\
\text { several days }\end{array}$ \\
\hline $\begin{array}{l}\text { 47. Premna esculenta Roxb. } \\
\text { Verbenaceae/ L5341: R; Fig. } 25\end{array}$ & $\begin{array}{l}\text { Silazra, Lelom } \\
\text { pada (C) } \\
\text { Laham shak } \\
\text { (M) }\end{array}$ & $\begin{array}{l}\text { Fungal/ } \\
\text { Bacterial } \\
\text { infections }\end{array}$ & & $\begin{array}{l}\text { Leaf-paste applied to } \\
\text { affected areas }\end{array}$ \\
\hline $\begin{array}{l}\text { 48. Saraca asoca (Roxb.) Wild. } \\
\text { Caesalpiniaceae/ L.2801a: R; } \\
\text { Fig. } 18\end{array}$ & $\begin{array}{l}\text { Moma gaas, } \\
\text { Paillang moma, } \\
\text { Kalamagma } \\
\text { (C) }\end{array}$ & Itching & Leaf & $\begin{array}{l}\text { Leaf extract applied } 2-3 \\
\text { times a day }\end{array}$ \\
\hline $\begin{array}{l}\text { 49. Sarcochlamys pulchirrima Gaud. } \\
\text { Urticaceae/ L1174: R }\end{array}$ & $\begin{array}{l}\text { Jangaillya shak } \\
\text { (C) }\end{array}$ & $\begin{array}{l}\text { Boils, } \\
\text { blisters } \\
\text { Eye- } \\
\text { itching }\end{array}$ & Leaf & $\begin{array}{l}\text { Leaf-paste applied to } \\
\text { affected areas; } \\
\text { fresh leaf extract applied } \\
\text { as eye drop }\end{array}$ \\
\hline $\begin{array}{l}\text { 50. Saurauia roxburghii Wall. } \\
\text { Actinidiaceae/ L3611: R }\end{array}$ & $\begin{array}{l}\text { Bhola kadam } \\
\text { (C) }\end{array}$ & Boils & Leaf & $\begin{array}{l}\text { Infusion of leaves used in } \\
\text { baths, specially for } \\
\text { children }\end{array}$ \\
\hline $\begin{array}{l}\text { 51. Senna alata (L.) Roxb. } \\
\text { Caesalpiniaceae/L3048a: R; Fig. } 5\end{array}$ & $\begin{array}{l}\text { Delong pata } \\
\text { (C) } \\
\text { puishibang }(\mathrm{M})\end{array}$ & Eczema & Leaf & $\begin{array}{l}\text { Leaf-paste applied twice } \\
\text { daily }\end{array}$ \\
\hline $\begin{array}{l}\text { 52. Spermacoce stricta L.f. } \\
\text { Rubiaceae/kher; L4489: R; Fig.14 }\end{array}$ & $\begin{array}{l}\text { Bishmijal, } \\
\text { Mijlick }\end{array}$ & Scabies & Leaf & $\begin{array}{l}\text { Leaf-paste is applied to } \\
\text { affected areas }\end{array}$ \\
\hline $\begin{array}{l}\text { 53. Tarenna campaniflora (Hook.f.) } \\
\text { N.P. Balakr. } \\
\text { Rubiaceae/ L3126: R; Fig. } 8\end{array}$ & $\begin{array}{l}\text { Rebekphul } \\
\text { gaas, Harulodi, } \\
\text { Barochallya } \\
\text { (C) }\end{array}$ & $\begin{array}{l}\text { a) eczema } \\
\text { b) scabies } \\
\text { c) warts }\end{array}$ & Leaf & $\begin{array}{l}\text { a) Leaf extract applied } \\
\text { externally b) infusion of } \\
\text { leaves used in baths c) } \\
\text { leaf-paste applied to } \\
\text { affected areas }\end{array}$ \\
\hline $\begin{array}{l}\text { 54. Trema orientalis (L.) Bl. } \\
\text { Ulmaceae/ L1204:K }\end{array}$ & $\begin{array}{l}\text { Sugarar amila } \\
\text { (T) }\end{array}$ & $\begin{array}{l}\text { Septic } \\
\text { abscess }\end{array}$ & Stem & $\begin{array}{l}\text { Stem-paste applied twice } \\
\text { daily }\end{array}$ \\
\hline $\begin{array}{l}\text { 55. Trichosanthes anguina L. } \\
\text { Cucurvitaceae/ L1737: K }\end{array}$ & $\begin{array}{l}\text { Horinkan shak, } \\
\text { Faranga ludi } \\
\text { (C) }\end{array}$ & Boils & Leaf & $\begin{array}{l}\text { Fresh lea-paste applied to } \\
\text { affected areas }\end{array}$ \\
\hline $\begin{array}{l}\text { 56. Uraria prunellaefolia Grah. } \\
\text { Papilionaceae/ L662a: R }\end{array}$ & $\begin{array}{l}\text { Belio lengur } \\
\text { (C) }\end{array}$ & Boils & Leaf & $\begin{array}{l}\text { Leaf-paste applied over } \\
\text { boils 2-3 times at night }\end{array}$ \\
\hline $\begin{array}{l}\text { 57. Vernonia patula (Dryand) Merr. } \\
\text { Asteraceae/L4519: R }\end{array}$ & Daduppon (C) & $\begin{array}{l}\text { Wounds, } \\
\text { Skin } \\
\text { burn }\end{array}$ & $\begin{array}{l}\text { Whole } \\
\text { plant }\end{array}$ & $\begin{array}{l}\text { Plant extract applied } \\
\text { twice daily }\end{array}$ \\
\hline $\begin{array}{l}\text { 58. Vitis pentagona (Roxb.) Lawson } \\
\text { Vitaceae/ L1713a: K; Fig. } 2\end{array}$ & $\begin{array}{l}\text { Hajjangludi } \\
\text { (C) }\end{array}$ & $\begin{array}{l}\text { Eczema, } \\
\text { infection }\end{array}$ & Leaf & $\begin{array}{l}\text { Leaf extract applied to } \\
\text { affected areas }\end{array}$ \\
\hline $\begin{array}{l}\text { 59. Xanthium indicum J. Koenig; } \\
\text { Asteraceae/ L3450a: R; Fig. } 6\end{array}$ & $\begin{array}{l}\text { Bengal lengera } \\
\text { (C) }\end{array}$ & $\begin{array}{l}\text { Infection } \\
\text { Of fingers }\end{array}$ & Leaf & $\begin{array}{l}\text { Leaf-paste applied to } \\
\text { affected areas }\end{array}$ \\
\hline $\begin{array}{l}\text { 60. Ziziphus oenoplia Mill. } \\
\text { Rhamnaceae/1938: R }\end{array}$ & Bon boroi $(\mathrm{T})$ & $\begin{array}{l}\text { Boils, } \\
\text { itching }\end{array}$ & Leaf & $\begin{array}{l}\text { Paste prepared from fresh } \\
\text { tender leaves applied } \\
\text { twice daily }\end{array}$ \\
\hline
\end{tabular}



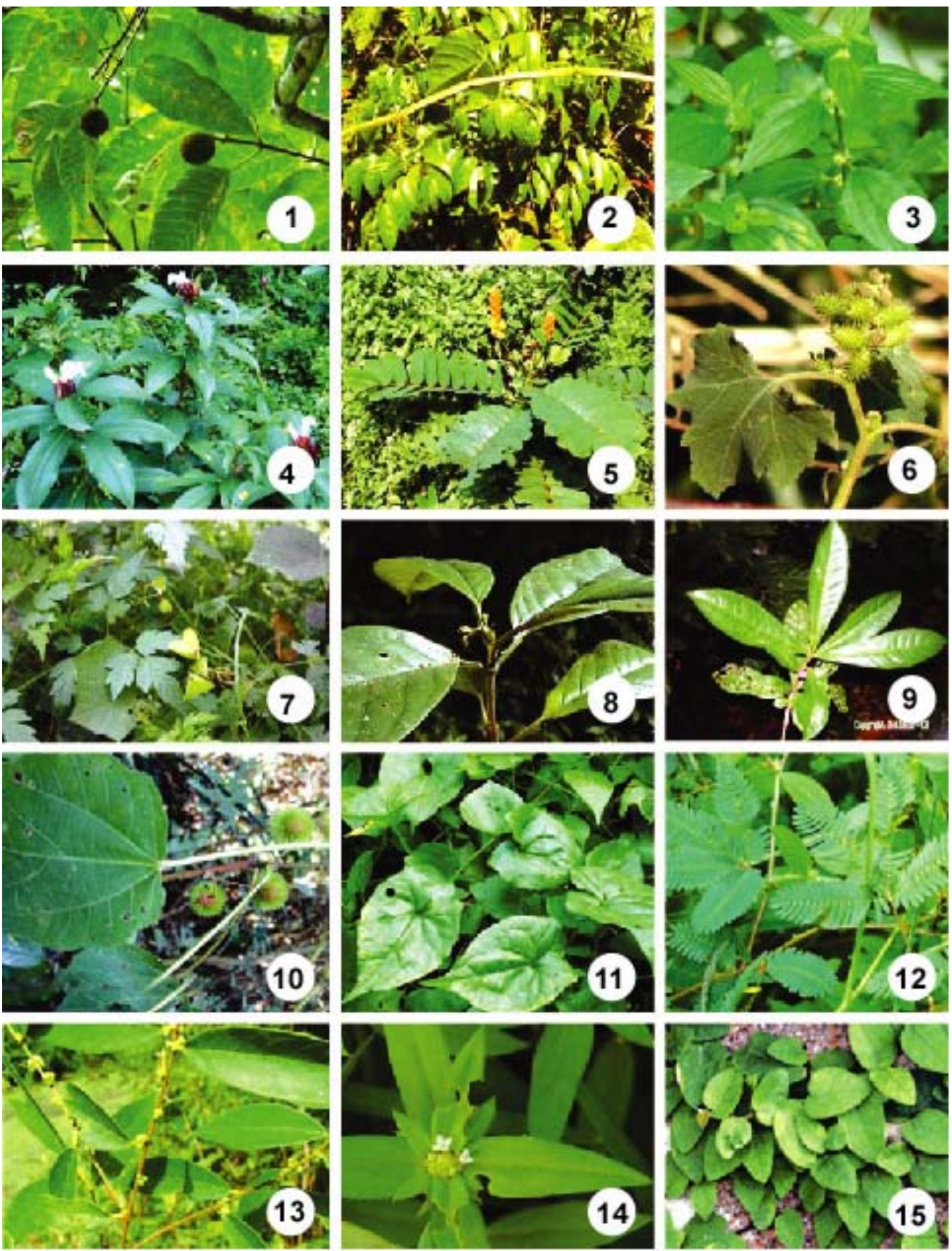

Figs 1-15. Habit of plants: 1. Neonauclea sessilifolia (Roxb.) Merr. 2. Vitis pentagona (Roxb.) Lawson. 3. Pouzolzia sanguinea (Blume) Merr. 4. Costus speciosus Miers. 5. Senna alata (L.) Roxb. 6. Xanthium indicum J. Koenig. 7. Cardiospermum helicacabum L. 8. Tarenna campaniflora (Hook. f.) Balakr. 9. Ardisia solanacea Roxb. 10. Byttneria pilosa Roxb. 11. Mikania micrantha Kunth. 12. Mimosa pudica L. 13. Bridelia stipularis (L.) Blume. 14. Spermacoce stricta L. f. 15. Ficus mollis Vahl. 

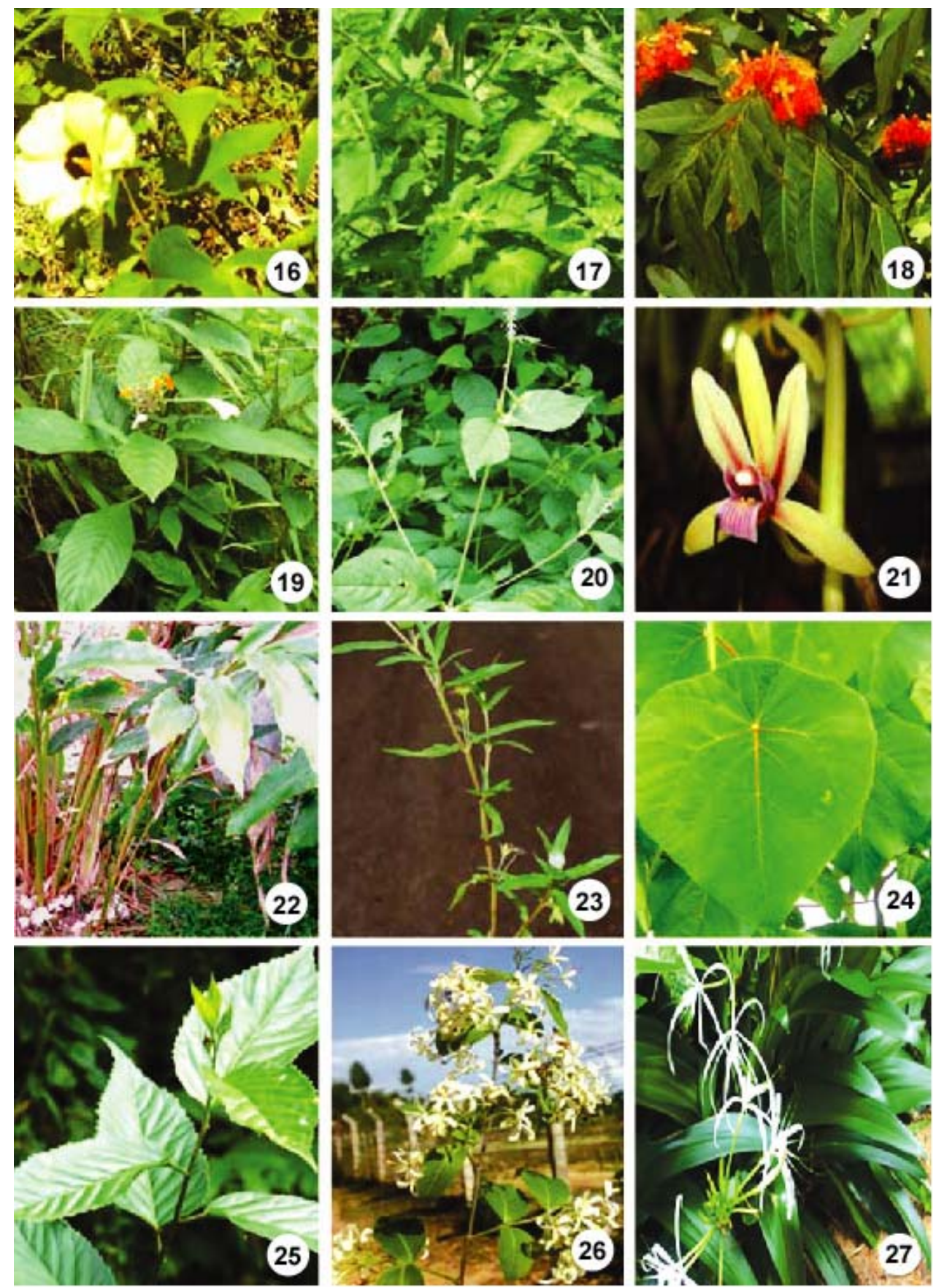

Figs 16-27. Habit of plants: 16. Abelmoschus moschatus Medik. 17. Hyptis suaveolens (L.) Poit. 18. Saraca asoca (Roxb.) Wild. 19. Mussaenda roxburghii Hook. f. 20. Achyranthes aspera L. 21. Cymbidium aloifolium (L.) Sw. 22. Amomum dealbatum Roxb. 23. Eclipta alba L. 24. Macaranga peltata (Roxb.) Merr. 25. Premna esculenta Roxb. 26. Holarrhena antidysenterica Wall. 27. Crinum asiaticum L. 


\section{References}

Ahmed ZU, ZNT Begum, MA Hassan, M Khondker, SMH Kabir, M Ahmad, ATA Ahmed, AKA Rahman and EU Haque (Eds) 2009. Encyclopedia of Flora and Fauna of Bangladesh. Vol. 9. Angiosperms (Magnoliaceae-Puncaceae). Asiat. Soc. Bangladesh, Dhaka.

Ahmed ZU, ZNT Begum, MA Hassan, M Khondker, SMH Kabir, M Ahmad, ATA Ahmed, AKA Rahman and EU Haque (Eds) 2009. Encyclopedia of Flora and Fauna of Bangladesh. Vol. 10. Angiosperms (Ranunculaceae-Zygophyllaceae). Asiat. Soc. Bangladesh, Dhaka.

Alam MK 1992. Medical ethnobotany of Marma Tribe of Bangladesh. Econ. Bot. 46(3): 330-335.

Alam MK, J Chowdhury and MA Hassan 1996. Some folk formularies from Bangladesh. J. Life Sci. 8(1): 49-63.

Chakma S 1992. Chakma Parichiti. Bangang Publication, Rangamati.

Chakma S 1993. Parbattay Chattagram-er Upajati O Sanskriti. Bangang Publication, Rangamati, Bangladesh.

Chopra RN 1958. Indigenous Drugs of India, 2nd edition, Art Press, Calcutta. 356 pp.

Chopra RN, SL Nayar and IC Chopra 1956. Glossary of Indian Medicinal Plants. CSIR, New Delhi.

Dastur JF 1977. Medicinal plants of India and Pakistan. DB Tarapore Vala Sons Co. Ltd., India.

Ghani A 2002. Medicinal Plants of Bangladesh with chemical constituents and uses. 2nd edition. Asiat. Soc. Bangladesh, Dhaka. 460 pp.

Hassan MA and AM Huq 1993. Amader Bonoushudi Shampad. Hassan Book House, Dhaka. 184 pp.

Hassan MA and MS Khan 1986. Ethnobotanical records in Bangladesh -1. Plants used for healing fractured bones. J. Asiat. Soc. Bangladesh (Sci.) 12(1-2): 33-39.

Hassan MA and MS Khan 1996. Ethnobotanical records in Bangladesh - 2. Plants used for healing cuts and wounds. Bangladesh J. Plant Taxon. 3(2): 49-52.

Jain SK 1991. Dictionary of Indian folk medicine and ethnobotany. Deep Publications, New Delhi.

Kapoor LD 1990. CRC Handbook of Ayurvedic medicinal plants. CRC Press, Inc. Boca Raton, Florida. $416 \mathrm{pp}$.

Khan MS, MH Hassan and MJ Uddin 2002. Ethnobotanical survey in Rema Kelanga Wildlife Sanctuary (Habiganj) in Bangladesh J. Plant Taxon. 9(1): 51-60.

Kirtikar CKR and BD Basu 1980. Indian medicinal plants. Bishen Singh Mahendra Pal Singh, Dehra Dun.

Mia MK and AM Huq 1988. A preliminary ethnobotanical survey in the Jaintapur, Tamabil and Jaflong area, Sylhet. Bull. 3: 1-10. Bangladesh National Herbarium, Dhaka.

Nadkarni KM 1982. Indian Materia Medica. Popular Proakashan, Bombay.

Rahman MA 1997. Tribal knowledge of plant use in Hill Tracts districts of Bangladesh. Biodiversity Newsletter Bangladesh 1(1): 1.

Rahman MA 2003. Ethno-medico-botanical knowledge among tribals of Bangladesh. In: Ethnobotany and medicinal plants of Indian subcontinent, Maheshwari, JK (Ed), pp. 89-93. Scientific Publisher, Jodhpur.

Rahman MA and SB Uddin 1998. Some anti-rheumaticplants used by tribal people of Hill Tracts districts of Bangladesh. Biodiversity Newsletter Bangladesh 2(2): 4.

Rahman MA, SB Uddin and A Khisha 1998. A report on some anti-jaundice plants from tribal communities of Hill Tracts districts of Bangladesh. Biodiversity Newsletter Bangladesh 2(1): 4.

Rahman MA, SB Uddin and CC Wilcock 2003a. Indigenous knowledge of herbal medicine in Bangladesh 1: for the cure of Jaundice. Hamdard Medicus 40(2): 25-28.

Rahman MA, SB Uddin and CC Wilcock 2003b. Indigenous knowledge of herbal medicine in Bangladesh 2: diarrhoea, dysentery, indigestion and stomach pains. J. Med. Arom. Pl. Sci. 25: 1001-1009.

Rahman MA, SB Uddin and CC Wilcock 2007. Medicinal plants used by Chakma tribe in Hill Tracts districts of Bangladesh. Indian J. Trad. Knowledge 6(3): 508-517.

Rahman MA, A Khisa, SB Uddin and CC Wilcock 2000. Indigenous knowledge of plant use in Hill Tracts tribal community and its role in sustainable development. In: Indegenous knowledge development in Bangladesh. Sillitoe P (Ed). pp. 75-78. The University Press Ltd., Dhaka. 
Yusuf M, MA Rahman, JU Chowdhury and J Begum 2003. Indigenous knowledge about the use of zingibers in Bangladesh J. Econ. Taxon. Bot. 26(3): 566-570.

Yusuf M, JU Chowdhury, MA Wahab and J Begum 1994. Medicinal pants of Bangladesh. BCSIR Dhaka. $340 \mathrm{pp}$.

Yusuf M, J Begum, MN Hoque and JU Chowdhury 2009. Medicinal pants of Bangladesh. BCSIR Chittagong. 794 pp.

(Manuscript received on 16 May, 2010; revised on 24 November, 2010) 